\title{
On the equivalence of regularization schemes *
}

\author{
Ji-Feng Yang \\ Department of Physics, East China Normal University, Shanghai 200062, P R China
}

\begin{abstract}
We illustrated via the sunset diagram that dimensional regularization 'deforms' the nonlocal contents of multi-loop diagrams with its equivalence to cutoff regularization scheme recovered only after sub-divergence were subtracted. Then we employed a differential equation approach for calculating loop diagrams to verify that dimensional regularization deformed the 'low energy' contents before subtraction. The virtues of the differential equation approach were argued especially in nonperturbative perspective.
\end{abstract}

*Work supported in part by the National Nature Science Foundation of China under Grant No. 10075020. 


\section{INTRODUCTION}

Recently, there have been intensive applications of the effective field theory method [1] to nucleon interactions [2]. In these applications UV divergences occur and are subtracted following the field theoretical renormalization principles. However, since the employed framework is in fact nonperturbative ones, there appear some subtle issues like the applicabilities of various regularization and/ or subtraction schemes [3]. It is recently shown that the dimensional regularization is equivalent to cutoff scheme in the only after it is combined with some nonminimal subtraction scheme [4].

In this report, we discuss the subtleties associated with the conventional regularization schemes. We wish to emphasize that dimensional regularization is inequivalent to cutoff regularization in parametrizing the nonlocal contents of multi-loop diagrams before any subtraction is done, and that the equivalence is only recovered with a sub-divergence subtraction that affected the definite nonlocal part, in contrast to the cutoff scheme situation. On the other hand the cutoff scheme is notorious for its power divergences. Although these facts are already known to high energy theorists, we still feel it necessary to point out that the renormalization is easily operable within the perturbative diagrammatic contexts, but not necessarily so in various (known and unknown) nonperturbative contexts. Thus we should be aware of this subtlety when working in nonperturbative contexts.

This report is organized as follows. In section two, we exhibit the obviously different results calculated respectively through dimensional and cutoff regularization. The difference in the leading definite part is removed after the sub-divergence is removed. Then, in section three we apply a differential equation approach to calculate the two loop integral and find that it is compatible with the cutoff result but not with the dimensional regularization result before subtraction. The last section is devoted to discussion and the summary. 


\section{THE SUNSET DIAGRAM IN MASSIVE $\lambda \phi^{4}$}

First let us specify the lagrangian

$$
L \equiv \frac{1}{2}(\partial \phi)^{2}-\frac{1}{2} m^{2} \phi^{2}-\frac{\lambda}{4 !} \phi^{4} .
$$

To focus on the mass dependence we put the external momentum to zero. The two loop self-energy diagram under consideration is

$$
\Sigma_{\theta}^{(2)}\left(0, m^{2}\right) \equiv-\frac{\lambda^{2}}{6} I_{\theta}\left(m^{2}\right) \equiv-\frac{\lambda^{2}}{6} \iint \frac{d^{4} k d^{4} l}{(2 \pi)^{8}} \frac{1}{\left(k^{2}+m^{2}\right)\left((k+l)^{2}+m^{2}\right)\left(l^{2}+m^{2}\right)}
$$

where we have Wick-rotated all the internal momentum integrals. This is a nontrivial two loop diagram with overlapping divergences. This diagram has been calculated a number of times in various regularization schemes [5 [7], we can extract from these papers the expressions for $I_{\theta}\left(m^{2}\right)$. First let us see the dimensional regularization result.

\section{A. Dimensional regularization}

From Ref. [6] we find that in $D$ dimensions

$$
I_{\theta}^{(D)}\left(m^{2}\right)=\frac{\Gamma(3-D)}{(4 \pi)^{2 D}} \int_{0}^{1}[d \alpha d \beta d \gamma] \delta(\alpha+\beta+\gamma-1) \frac{\left(m^{2}(\alpha+\beta+\gamma)\right)^{D-3}}{(\alpha \beta+\beta \gamma+\gamma \alpha)^{D / 2}},
$$

and from [7] we have

$$
\begin{aligned}
& I_{\theta}^{(D)}\left(m^{2}\right)=-\frac{2 \Gamma(2-D / 2) \Gamma(1-D / 2) m^{2 D-6}}{(4 \pi)^{D}} X(1), \\
& X(1) \equiv \frac{2}{3(3-D)}+3^{(D-3) / 2} \int_{0}^{1} d u \frac{u^{2-D / 2} /(4-u)-1}{u^{1 / 2}(4-u)^{(D-1) / 2}} .
\end{aligned}
$$

The full $\epsilon$-expansion is given in [7] as

$$
(4 \pi)^{4} I_{\theta}^{(D)}\left(m^{2}\right)=-\frac{3 m^{2}}{2 \epsilon^{2}}\left(1+(3-2 \bar{L}(m)) \epsilon+a \epsilon^{2}+\cdots\right)
$$

with $\bar{L}(x) \equiv \ln \left(x^{2} / \mu^{2}\right)+\gamma_{E}-\ln 4 \pi, \gamma_{E}$ Euler's constant and

$$
\left.a=2 \bar{L}^{2}(m)-6 \bar{L}(m)\right)+7+\zeta(2)+\frac{1}{\sqrt{3}} \int_{0}^{\pi / 6} d x \ln (2 \sin x) .
$$


This result is the same as Collins' after carrying out the integrals in Eq.(3)

$$
(4 \pi)^{4} I_{\theta}^{(D)}\left(m^{2}\right)=-\frac{3 m^{2}}{2}\left\{\frac{1}{\epsilon^{2}}+\frac{3-2 \bar{L}(m)}{\epsilon}-6 \bar{L}(m)+2 \bar{L}^{2}(m)+\text { const. }\right\} .
$$

Now we turn to the cutoff scheme.

\section{B. Cutoff regularization}

This two loop integral calculated in cutoff scheme can be extracted from Ref. [5]

$$
(4 \pi)^{4} I_{\theta}^{(\Lambda)}\left(m^{2}\right)=-\frac{3 m^{2}}{2}\left\{\ln ^{2}\left(m^{2} / \Lambda^{2}\right)+2 \ln \left(m^{2} / \Lambda^{2}\right)\right\}+C \Lambda^{2}
$$

with the coefficient $C$ of $\Lambda^{2}$ term not explicitly given there. Through direct calculation $C$ can be determined as $C=2$.

In order to make the comparison easier, we cast the cutoff result into the following form

$$
(4 \pi)^{4} I_{\theta}^{(\Lambda)}\left(m^{2}\right)=\frac{-3 m^{2}}{2}\left\{\bar{L}^{2}(\Lambda)+\bar{L}(\Lambda)(2-2 \bar{L}(m))-2 \bar{L}(m)+\bar{L}^{2}(m)\right\}+2 \Lambda^{2} .
$$

\section{Disadvantages of conventional regularization schemes}

Now it is clear that the leading finite term (a double $\log \bar{L}^{2}(m)$ ) that has different coeffi-

cients in different schemes, i.e., $-\frac{3 m^{2}}{(4 \pi)^{4}} \bar{L}^{2}(m)$ in dimensional regularization and $-\frac{3 m^{2}}{2(4 \pi)^{4}} \bar{L}^{2}(m)$ in cutoff scheme. This double log is the leading definite part of the sunset diagram that should be independent of the UV regularizations residing in the local part. Thus subtraction of divergence (including sub-divergence) should not alter the leading finite terms(nonlocal), or the leading finite term is renormalization/subtraction scheme independent. But in dimensional scheme, the sub-divergence subtraction does affect this double log as can be seen from the counter-term in Ref. [7]:

$$
\begin{aligned}
& I_{\theta}^{(D) \prime}\left(m^{2}\right) \equiv I_{\theta}^{(D)}\left(m^{2}\right)-\frac{3}{(4 \pi)^{2} \epsilon} I_{1}^{(D)}\left(m^{2}\right) \\
= & I_{\theta}^{(D)}\left(m^{2}\right)+\frac{3}{(4 \pi)^{4} \epsilon^{2}}\left\{1+(1-\bar{L}(m)) \epsilon+\left(\frac{\bar{L}^{2}(m)}{2}-\bar{L}(m)+1+\frac{\zeta(2)}{2}\right) \epsilon^{2}+\cdots\right\} \\
= & -\frac{3 m^{2}}{2(4 \pi)^{4} \epsilon^{2}}\left\{-1+\epsilon+\left(\bar{L}^{2}(m)-4 \bar{L}(m)\right) \epsilon^{2}+\cdots\right\}
\end{aligned}
$$


where $I_{1}^{(D)}\left(m^{2}\right) \equiv \int \frac{\mu^{2 \epsilon} d^{D} k}{(2 \pi)^{D}} \frac{1}{k^{2}+\Omega^{2}}$ denotes the tadpole sub-diagram. It is obvious that the leading double log now is in agreement with cutoff scheme result as the cutoff scheme subdivergence subtraction will not affect the double $\log$ at all. It is also obvious that the counter-term did subtract the leading nonlocal term in dimensional regularization.

The origin of the problem lies in the deformation of the low energy (nonlocal) content of a diagram in the dimensional regularization that should be independent of regularization schemes as it is purely 'low energy' part that should be unaffected by the 'high energy' details. Although we can remove it through sub-diagram subtractions within perturbative context, we feel that it is a subtle and disadvantageous aspect of dimensional regularization that might cause problems in other more complicated contexts than the perturbative one.

One might argue in favor of dimensional regularization that there is no notorious quadratic and quartic divergences in it. In our opinion, the vanishing of power form divergences in dimensional regularization might be understood as an implicitly built-in subtraction, while such subtraction must be explicitly done in cutoff scheme. It is more natural to prefer the ability to produce the correct nonlocal (or low energy) information. However, more explicit divergences did make their removal more difficult in nonperturbative contexts. It is demanding to find a scheme to get rid of these weak points. This is what we wish to do in next section. In addition, we mention that in dimensional scheme the definition of metric tensor and the Dirac algebra becomes a rather nontrivial and complicated task, especially in presence of fermions.

\section{A DIFFERENTIAL EQUATION ANALYSIS OF LOOP DIAGRAMS}

Now let us calculate the diagram by adopting the standard point of view that all the known QFT's are effective theories for a completely well-defined quantum theory containing

all the underlying high energy details [8]. Since we have not found the complete theory yet, usually we introduce some artificial deformations to make QFT's UV finite that might be physically inappropriate, for example dimensional regularization deforms the low energy 
parts that must be recovered through subtraction in the perturbative context.

However, it is well known fact that differentiation with respect to physical parameters (usually external momenta and/or masses) reduces the divergence degrees (or more rigorously the ill-definedness degrees) of the divergent diagrams [9]. We can perform the differentiation on a diagram $\gamma$ for enough times (for $\omega_{\gamma}+1$ times with the divergence degree $\omega_{\gamma}$ ) to yield a sum of diagrams without any superficial ill-definedness. If there is still sub-divergence, repeat the operation on the sub-diagrams till no ill-definedness is left so that the loop integrals can be safely carried out. Finally we integrate back with respect to the momenta (and/or masses) 'external' to the loop treated, which can be done diagram by diagram and loop by loop [10].

Technically, this approach amounts to calculating the ill-defined loop diagrams by solving of well defined differential equations derived from the existence of the underlying theory and its 'low energy' limits, a generalization of the WT identity in gauge theories. The ill-defined diagrams or loops should be well defined in the underlying theory with the ill-definedness indicating the lacking of necessary UV details in the effective theories or the incompleteness of the effective theories. Then in this approach the solutions would naturally contain unknown constants parametrizing the ill-definedness or incompleteness (to be fixed by physical 'boundary conditions') in contrast to the cutoff and dimensional schemes where ill-definedness is parametrized in terms of divergences, which is an eminent progress in regularization. It is obvious that this approach needs neither artificial deformations nor unnatural cutoffs. And the metric tensor and Dirac algebra need no more additional treatment here.

Now we apply this method to the diagram discussed in last section, that is to determine the two loop integral $I_{\theta}\left(m^{2}\right)$.

(1). First, we differentiate it with respect to mass square $\left(m^{2}\right)$ for two times (differentiation with respect to momentum could achieve the same goal), that is,

$$
\begin{aligned}
\frac{\partial^{2}}{\partial_{\left(m^{2}\right)^{2}}} I_{\theta}\left(m^{2}\right) & \equiv 6 I_{\theta:(3 ; 1 ; 1)}\left(m^{2}\right)+3 I_{\theta:(2 ; 2 ; 1)}\left(m^{2}\right)+3 I_{\theta:(2 ; 1 ; 2)}\left(m^{2}\right), \\
I_{\theta:(\alpha ; \beta ; \gamma)}\left(m^{2}\right) & \equiv \int \frac{d^{4} k d^{4} l}{(2 \pi)^{8}\left(k^{2}+m^{2}\right)^{\alpha}\left((k+l)^{2}+m^{2}\right)^{\beta}\left(l^{2}+m^{2}\right)^{\gamma}} .
\end{aligned}
$$


The result is a sum of three new diagrams without any overall divergence. Among these diagrams, $I_{\theta:(2 ; 2 ; 1)}\left(m^{2}\right)$ and $I_{\theta:(2 ; 1 ; 2)}\left(m^{2}\right)$ contain no sub-divergence while $I_{\theta:(3 ; 1 ; 1)}\left(m^{2}\right)$ contains a sub-divergence in the integration of loop momentum $l$

$$
I_{(1 ; 1)}\left(m^{2}, k^{2}\right) \equiv \int \frac{d^{4} l}{(2 \pi)^{4}\left((k+l)^{2}+m^{2}\right)\left(l^{2}+m^{2}\right)} .
$$

(2). Second, we treat this divergent sub-diagram with the same method described above which lead to the following inhomogeneous differential equation

$$
\begin{aligned}
\partial_{m^{2}} I_{(1 ; 1)}\left(m^{2}, k^{2}\right) & =-\int \frac{d^{4} l}{(2 \pi)^{4}} \frac{\left((k+l)^{2}+m^{2}\right)+\left(l^{2}+m^{2}\right)}{\left((k+l)^{2}+m^{2}\right)^{2}\left(l^{2}+m^{2}\right)^{2}} \\
& =\frac{-1}{(4 \pi)^{2}} \int_{0}^{1} \frac{d x}{m^{2}+\left(x-x^{2}\right) k^{2}} .
\end{aligned}
$$

The solution to this equation is easy to find,

$$
I_{(1 ; 1)}\left(m^{2}, k^{2}\right)=\frac{-1}{(4 \pi)^{2}} \int_{0}^{1} d x\left\{\ln \left(m^{2}+\left(x-x^{2}\right) k^{2}\right)+c_{(1: 1)}\right\}
$$

with $c_{(1 ; 1)}$ being the integration constants independent of any physical parameters like masses and momenta. In specific regularization schemes, $c_{(1 ; 1)}$ are taken place by various constants containing divergent ones, e.g., $\ln \Lambda^{2}$ in cutoff scheme. In principle, it should be fixed by 'boundary condition'.

(3). Now we can compute the right hand side of Eq.(11) and obtain again an inhomogeneous differential equation as below

$$
\frac{\partial^{2}}{\partial_{\left(m^{2}\right)^{2}}} I_{\theta}\left(m^{2}\right)=-\frac{3}{(4 \pi)^{4}} \frac{\ln m^{2}+c_{(1 ; 1)}-1}{m^{2}}
$$

and the solution to it reads

$$
I_{\theta}\left(m^{2}\right)=-\frac{3 m^{2}}{2(4 \pi)^{4}}\left\{\ln ^{2} m^{2}+2\left(c_{(1 ; 1)}-2\right) \ln m^{2}-2 c_{(1 ; 1)}+4+2 c_{\theta ; 1}\right\}-\frac{3 c_{\theta ; 2}}{(4 \pi)^{4}}
$$

with $c_{\theta ; 1}, c_{\theta ; 2}$ being the constants (independent of masses, coupling and momenta) to be fixed by 'boundary conditions', as pointed out in the preceding paragraghs.

It is clear that the leading double $\log \left(-\frac{3 m^{2}}{2(4 \pi)^{4}} \ln ^{2} m^{2}\right)$ determined from the differential equation Eq.(16) naturally satisfied by $I_{\theta}\left(m^{2}\right)$ agrees with that determined from cutoff 
scheme but disagrees with that from dimensional regularization. In fact we can define the constants $c_{(1 ; 1)}, c_{\theta ; 1}$ and $c_{\theta ; 2}$ in such a way that the cutoff result is exactly reproduced, i.e., the cutoff scheme can be a particular solution to the differential equations while dimensional regularization is not. Thus, dimensional regularization is disfavored in the sense just specified. We stress again that this is a property before subtraction.

As was already mentioned above, dimensional regularization 'deforms' the low energy information of a quantum theory that must be recovered through a necessary subtraction of some low energy contents, while the cutoff scheme preserves the low energy information faithfully but yields some ugly divergences. In this connection, our differential equation approach is more favorable. It should show particular advantage in nonperturbative contexts since the troublesome work of subtracting divergences (perturbative and/or nonperturbative) is replaced by the easier work of fixing the undetermined constants through physical 'boundary conditions'. Moreover, we need not the extra recourse to the tricky procedures for manipulating infinities and the intermediate renormalized 'quantities' that must be finally translated into physical 'quantities'. It is not difficult to see that in certain nonperturbative problems, to parameterize the ill-definedness in terms of nonperturbative divergences might make the subtraction procedure extremely complicated and awkward might even lead to no useful (or trustworthy) predictions which is physically unfavorable, while to parametrize the ill-definedness in terms of unknown constants will incur no awkwardness and make physical predictions more available [11], a superiority over the conventional schemes.

\section{DISCUSSIONS AND SUMMARY}

Where is the technical source of the subtlety for dimensional regularization? We feel that it is in the special way that dimensional regularization parameterizes the finite nonlocal piece, it is in the functional form of $\left(m^{2} \text { or } p^{2}\right)^{-n \epsilon}$ and its expansion in terms of $\epsilon$ in the $n$-loop integrals. It is mathematically sound to expand $\left(m^{2}\right)^{-2 \epsilon}$ as 


$$
\left(m^{2}\right)^{-2 \epsilon}=\exp \left(-2 \epsilon \ln m^{2}\right)=\sum_{n=0}^{\infty} \frac{(-2 \epsilon)^{n}}{n !}=1-2 \epsilon \ln m^{2}+2 \epsilon^{2} \ln ^{2} m^{2}+o\left(\epsilon^{3}\right) .
$$

But the $\epsilon^{2}$ order term has a 'incorrect' numeric coefficient 2. The double log should vanish in the tadpole diagram when the latter is not used to subtract the sub-divergences in the two-loop diagram, but should be kept when used for removing sub-divergence.

If one took the $\left(m^{2}\right)^{-2 \epsilon}$ as a product of two $\left(m^{2}\right)^{-\epsilon}$ with all higher order $\left(\epsilon^{n}, n \geq 2\right)$ terms dropped, one would get the correct double log, i.e.,

$$
\left(m^{2}\right)^{-2 \epsilon} \equiv\left\{\left(m^{2}\right)^{-\epsilon}\right\}^{2} \equiv\left\{1-\epsilon \ln m^{2}\right\}^{2}=1-2 \epsilon \ln m^{2}+\epsilon^{2} \ln ^{2} m^{2} .
$$

Unfortunately, this 'one-loop' convention has at least two problems: (a). One has to specify how to expand other factors that are functions of $\epsilon$ in a manner that is consistent with this 'one-loop' convention, and one can find that there can be several ways to expand the factors that will yield different numerical constants; (b). The more serious problem is that when one makes dimensional regularization and cutoff scheme agree with each other on the double $\log$ in this two loop diagram, they will disagree with each other on other parts and on the other diagrams (and the subtraction of sub-divergence must be carefully defined).

Although the problem is analyzed in a perturbative example, our main concern is in the difficulties one might meet in nonperturbative contexts. Since nonperturbative investigations in standard model and symmetry breaking physics are inevitable, we feel it necessary to be aware of such regularization subtleties and to work out a more efficient scheme. We hope our discussions here might of some value both in the pure theoretical perspective and in the application perspective.

In summary, the subtleties in the regularization scheme equivalence was discussed through the sunset diagram in massive $\lambda \phi^{4}$ theory. We introduced a natural differential equation approach for calculating the loop diagrams to help the analysis and demonstrated that the widely used dimensional regularization deformed the 'low energy' contents of theory before subtraction is made in contrast to the cutoff scheme. Our differential equation approach showed more virtues comparing to the two widely used regularization schemes. 


\section{ACKNOWLEDGEMENT}

The author is grateful to W. Zhu for helpful conversations. 


\section{REFERENCES}

[1] S. Weinberg, Phys. Lett. B 91, 51 (1980); L. J. Hall, Nucl. Phys. B178, 75 (1981); B. Ovrut and H. Schnitzer, Nucl. Phys. B179, 381 (1981), B189, 509(1981).

[2] For recent reviews of applications in nuclear physics, please see, e.g., U. van Kolck, Prog. Part. Nucl. Phys. V43, 337 (1999) (Report No. nucl-th/9902015) and S. R. Beane et al, Report No. nucl-th/0008064.

[3] See, for example, the recent discussion over the use of regularization and renormalization schemes in the EFT treatment of nucleons interation: T.D. Cohen, Phys. Rev. C 55, 67(1997); D. R. Phillips, S. R. Beane and T. D. Cohen, Ann. Phys. 263, 255 (1998) (Report No. hep-th/9706070); S. R. Beane, T. D. Cohen and D. R. Phillips, Nucl. Phys. A632, 445 (1998); D. B. Kaplan, M. Savage and M. B. Wise, Phys. Lett. B424, 390 (1998); J. V. Steele and R. J. Furnstahl, Nucl. Phys. A637, 46 (1998); J. Gegelia, Phys. Lett. B429, 227 (1998); D. R. Phillips, S. R. Beane and M. C. Birse, Report No. nucl-th/9810049.

[4] D. R, Phillips, S. R. Beane and M. C. Birse, Report No. nucl-th/97810049 and references therein.

[5] R. Jackiw, Phy. Rev. D 9, 1686 (1974).

[6] J. C. Collins, Phys. Rev. D10, 1213 (1974).

[7] C. Ford and D. R. T. Jones, Phys. Lett. B274, 409 (1992).

[8] See, e.g., M. E. Peskin and D. V. Schroeder, An Introduction to Quantum Field Theory, (Addison-Wesley, 1995), Chapter 8 and S. Weinberg, The Quantum Theory of Fields, Volume I, (Cambridge University Press, Cambridge, 1995), Chapter 12. The author is grateful to Professor J. Polchinski for this information.

[9] W. E. Caswell and A. D. Kennedy, Phys. Rev. D 25, 392 (1982). 
[10] Ji-Feng Yang, Report No. hep-th/9708104; invited talk, in: Proceedings of the XIth International Conference 'Problems of Quantum Field Theory', Eds. B. M. Barbashov, G. V. Efimov and A. V. Efremov (Publishing Department of JINR, Dubna, 1999)(hepth/9901138); hep-th/0008037.

[11] For a concrete nonperturbative example, please cf. S.-y. Lou and G.-j. Ni, Phys. Rev. D40, 3040 (1989); G.-j. Ni, et al, Science in China A41, 1206 (1998); S.-S. Feng and G.-j. Ni, Int. J. Mod. Phys. A14, 4259 (1999). 